\title{
Surgical management of multiple uterine tumours in a dog
}

\begin{abstract}
A female GSD of 12 years age and weighing $25 \mathrm{~kg}$ was presented to the institute Referral Veterinary Polyclinics with the history of blood mixed pus like discharge through vagina, anorexia and distended abdomen. Clinical examination revealed animal appeared dull and depressed; pale mucous membrane with the distended abdomen. Biochemical parameters were within the normal range. Radiological examination revealed radiodensity in dorsocaudal area of the abdomen. Based on the clinical and radiological examination, the case was tentatively diagnosed as pyometra. On mid-ventral laparotomy under atropine-xylazine-ketamine anesthesia, multiple tumours were observed in the uterus. Ovariohysterectomy along with excision of tumour mass was performed. Abdominal muscles and skin were sutured in routine fashion. Histopathologically it was confirmed as uterine leiomyomas
\end{abstract}

Keywords: multiple tumours, uterine leiomyomas, dog
Volume 5 Issue 2 - 2018

\section{Divya Mohan, Swapan Kumar Maiti, Shivaraju S, Ramith KR, Sangeetha P, Bindhuja BV, Naveen Kumar \\ Division of Surgery, Indian Veterinary Research Institute, India}

Correspondence: Swapan Kumar Maiti, Principal Scientist, Surgery Division, Indian Veterinary Research Institute, Izatnagar-243 I22, Uttar Pradesh, India,Tel +94| 2049|69, Fax +91 581 2303284, Email swapanivri@gmail.com

Received: December 20, 2017 | Published: March 29, 2018
Abbreviations: GSD, German shepherd; Kg, kilograms; $\%$, percentage; IVRI, Indian veterinary research institute; $\mathrm{S} / \mathrm{c}$, subcutaneous; I/m, intramuscular; I/V, intravascular; $\mathrm{Mg}$, milligram; a, at the rate of

\section{Introduction}

Uterine neoplasms are rare in canines and it accounts for less than $0.5 \%$ reproductive tract tumours. ${ }^{1}$ The neoplasms of the female tubular tract are almost $3 \%$ of all canine tumours and among that $85-90 \%$ occur in vagina and vulva. ${ }^{2}$ The commonly reported histological type of tumours of the uterus is leiomyoma i.e 85 to 95 percent uterine tumors are benign (leiomyoma) and 10 percent are malignant (leiomyosarcomas). ${ }^{3}$ Leiomyoma is benign; slow growing, non-invasive, non-metastatic arising from smooth muscles of hollow organs. ${ }^{4}$ Steroid hormones, such as estrogens play a role in the pathogenesis of leiomyomas because it is observed that dogs with genital leiomyomas also have follicular cysts, estrogen-producing tumours, endometrial hyperplasia and mammary neoplasia. ${ }^{4}$ Clinical signs of the condition depend on the location, size of a tumour and also on the metastasis and sometimes accompanied with pyometra. ${ }^{5}$ In uterine leiomyoma symptoms includes abdominal distension accompanied with palpable abdominal mass, polydipsia, polyuria, anorexia and weight loss, vomiting, vaginal discharge, ascites and constipation. ${ }^{6}$ Abdominal radiography, ultrasonography can be used for diagnosis. Histopathology of the tumour mass is best in confirmation of the diagnosis.

\section{Case report}

This clinical observation was made at Institute referral veterinary polyclinics. A 12 years old female GSD weighing $25 \mathrm{~kg}$ was presented with a history of blood mixed with pus- like discharge from the vulva for the past 2 weeks. Owners reported that animal showing loss of appetite and it was operated for a mammary tumour one year back. Clinical examination revealed that animal was dull, depressed with pale mucous membrane and distended abdomen. Biochemical parameters were within the normal range. On radiographic examination, radiodensity was observed in the dorsocaudal area of the abdomen. Based on the clinical and radiological examination the case was tentatively diagnosed as pyometra. Following this, a decision was made to perform mid ventral celiotomy with the consent of the owner. It was advised to fast the animal for 12 hours and withdraw water for 3 hours prior to surgery. The animal was given atropine@ $0.045 \mathrm{mg} / \mathrm{kg}$ $\mathrm{s} / \mathrm{c}$, xylazine@1mg/kg i/v as preanaesthetic. Anaesthesia was induced with ketamine@5mg/kg i/v. Animal was given ceftriaxone@25mg/ $\mathrm{kg} \mathrm{i} / \mathrm{v}$ preoperatively. The surgical site was scrubbed and prepared for aseptic surgery. A caudal mid ventral celiotomy was performed, the uterus was exteriorized, and multiple tumours were observed in the uterus. Tumour mass along with the uterus was removed (Figure 1A) (Figure 1B); both the ovaries were also removed. Muscles were sutured in continuous pattern using vicryl size 1; the skin was opposed in interrupted pattern using polyamide (Figure 2). The animal was given antibiotics and anti-inflammatory for five days. Fluid therapy was given for two days, thereafter animal started taking food normally. Skin sutures were removed after 12 days. The tumour sample (Figure 3) was sent for histopathological examination and it was confirmed as leiomyoma (Figure 4).

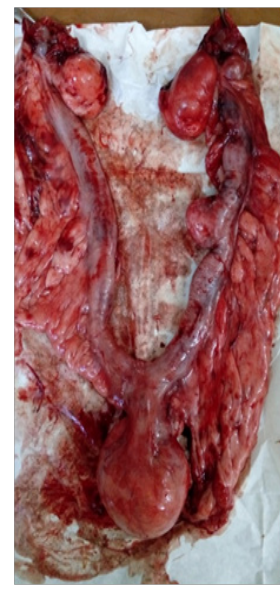

A

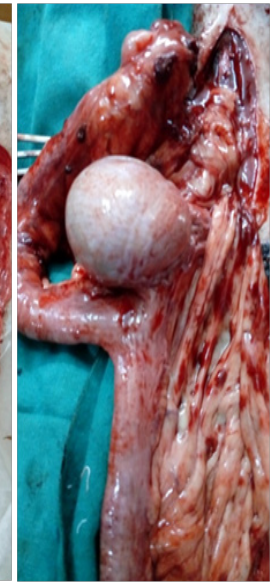

B
Figure I (A\&B) Multiple tumours in uterus. 


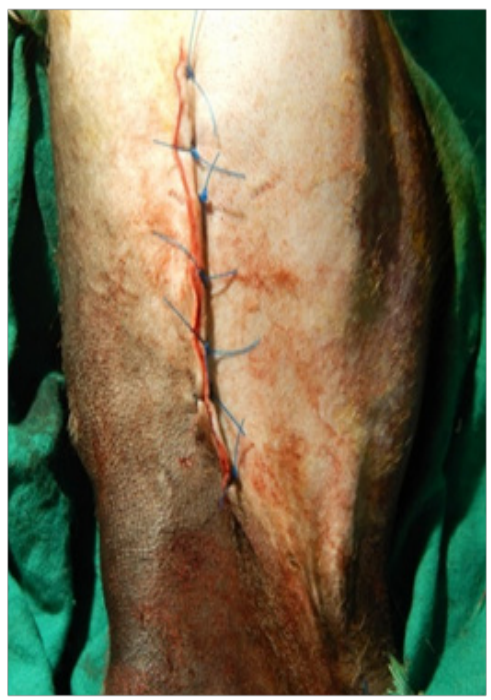

Figure 2 Skin suture after surgical intervention.

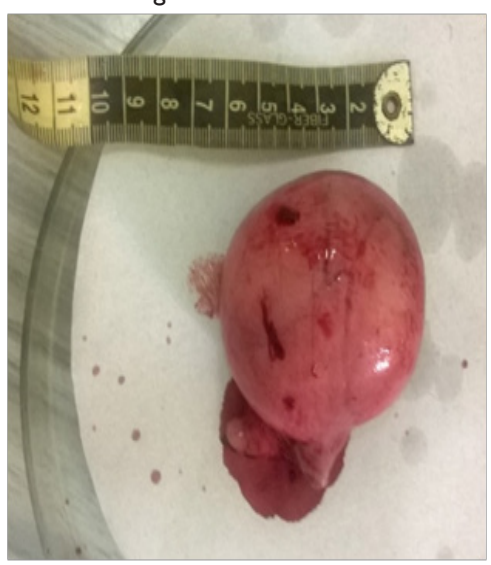

Figure 3 Uterine tumour mass.

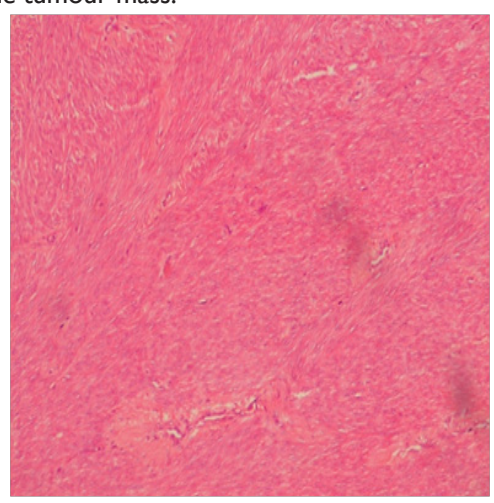

Figure 4 Leiomyoma (H\&E, IOX).

\section{Discussion}

Uterine tumours are rare in dogs and are incidental findings during postmortem or Ovariohysterectomy. ${ }^{4}$ Leiomyoma is a benign tumor arising from smooth muscle of hollow organs viz. uterus, vagina, intestines, stomach, urinary bladder and esophagus. ${ }^{7}$ Leiomyomas are the most common uterine tumors accounting for about $85-90 \%$ of all canine uterine tumours and are usually multiple. ${ }^{8}$ These are often associated with estrogen secreting tumours, follicular cyst, mammary hyperplasia or mammary neoplasia. ${ }^{4}$ In this case, the animal had also a history of a mammary tumour. Clinical signs usually arise with tumor mass pressing on abdominal organs leading to abdominal distension, anorexia, concurrent illness including pyometra. Uterine tumor enlargement may lead to obstruction of cervix and it further causes pyometra. ${ }^{9}$ Uterine tumours enlargement causes irritation, vascular erosion that leads to bloody discharge from the vulva. ${ }^{10}$ Ultrasonography or radiography is used for diagnosis but confirmation is possible by histopathological examination of tumour mass. ${ }^{11}$ Ovariohysterectomy is best curative for uterine tumours if there is no metastasis. ${ }^{9}$

\section{Conclusion}

In the present case, the clinical signs were not specific for the condition. The animal made uneventful recovery after pan hysterectomy along with excision of tumor mass. No metastasis was reported postoperatively.

\section{Acknowledgements}

The authors are highly thankful to Director, Indian Veterinary Research Institute (IVRI), Uttar-Pradesh, India providing necessary facilities to carry out the present work and Dr Pawan Kumar, Scientist, Pathology Division, IVRI for assisting histopathological works of this study.

\section{Conflict of interest}

There is no conflict of interest among the authors for publication of this article.

\section{References}

1. Brodey RS, Rozzel JF. Neoplasms of canine uterus, vagina and vulva. A clinico-pathologic survey of 90 cases. J Am Vet Med Assoc. 1967;151(10):1294-1307.

2. Karunakaran S, Pillai UN, Kuriakose AM, et al. Uterine leiomyoma in Rottweiler dog-A case report. Indian J of Vet Path. 2013;37(1):93-94.

3. Shammi M, Simon MS, Raj HP, et al. Surgical management of uterine leiomyoma in a bitch. Tamilnadu J Veterinary \& Animal Sciences. 2010;6(4):181-182.

4. Klein MK. Tumors of the female reproductive system. In: Withrow SJ, McEwen EG, editors. Small animal clinical oncology. 3rd ed. Philadelphia, USA: Saunders; 2001:445-454.

5. Murphy ST, Kruger JM, Watson GL. Uterine adenocarcinoma in the dog: a case report and review. J Am Anim Hosp Assoc. 1994;30:440-444.

6. McEntee MC. Reproductive oncology. Clin Tech Small Anim Pract. 2002;17(3):133-149.

7. Sastry GA. Veterinary Pathology. Neoplasms. Delhi: CBS publishers and distributors; 1983:232-233.

8. Karagiannis GS, Pelekanis M, Loukopoulos P, et al. Canine uterine leiomyoma with epithelial tissue foci, adenomyosis, and cystic endometrial hyperplasia. Case Reports in Veterinary Medicine. 2011;4.

9. White AS. Tumors of the genital tract. Manual of Small Animal Oncology. BSAVA. 1991;304.

10. Roberts JS. Veterinary Obstetrics and Genital Diseases. Infertility in cows. Delhi: CBS publishers and distributors; 2004: 488-489.

11. Harvey M. Conditions of the non-pregnant female. In: England G, Harvey $\mathrm{M}$, editors. Manual of small animal reproduction and neonatology. 1st ed. Quedgeley, Gloucester, UK: BSAVA; 1998:49. 